\title{
Thermodynamics Control Reactivity of Hypervalent Iodine-Mediated Styrene Hetero- and Homodimerization: Mechanistic Insights
}

Aqeel A. Hussein, ${ }^{1,2} *$ Ahmed Al-Yasari ${ }^{3,4}$ and Yumiao $\mathrm{Ma}^{5}$

\author{
${ }^{1}$ School of Chemistry, University of Southampton, Southampton, Hampshire, SO17 1BJ, United Kingdom. \\ ${ }^{2}$ College of Dentistry, University of Al-Ameed, Karbala PO Box 198, Iraq. \\ ${ }^{3}$ School of Chemistry, University of East Anglia, Norwich, NR4 7TJ, United Kingdom. \\ ${ }^{4}$ Department of Chemistry, Faculty of Sciences, University of Kerbala, Kerbala, Iraq. \\ ${ }^{5}$ BSJ Institute, Haidian, Beijing, 100084, People's Republic of China.
}

*Email: aahh1f19@ soton.ac.uk

\begin{abstract}
A mechanistic insight into the hetero- and homodimerizations (HETD and HOMD) of styrenes promoted by hypervalent iodine reagents (HVIRs; DMP and PIDA) and facilitated by HFIP to yield all trans cyclobutanes is reported using density functional theory (DFT) calculations. The HFIP molecules lower the energy of the single electron oxidation (SEO) or initiation as a result of strong hydrogen bonding interactions that substantially stabilize the frontier orbitals before and after electron addition. The HETD or HOMD is a radicallycharacterized $\pi-\pi$ stacked head-to-head stepwise [2+2] cycloaddition initiated via SEO by DMP or PIDA, respectively. DFT results supported by quasiclassical molecular dynamics simulations show that HOMD is a competing pathway to HETD although the latter is relatively faster, in accordance with experimental observations. The initiation is a rate-determining step as a thermodynamically endergonic and propagation is accomplished by radically-cationic hetero- and homodimerized intermediate as propagation is faster than single electron reduction (SER) or termination by radically-anionic HVIRs. Initiation by DMP found to be faster and less endergonic than by PIDA due to (1) the energy gap of electron transfer in a SEO step by I(V) is lower than I(III) and (2) the SOMO energy of the radical anion I(V) is lower than I(III). Furthermore, the presence of $p$ methoxy group is essential to underpin the SEO by which the more thermodynamically favorable SEO leads to a successful cycloaddition as the thermodynamic term represents a major contribution in the initiative barrier.
\end{abstract}

\section{Introduction}

Stereoselective approaches to substituted cyclobutanes have been captivated by organic chemists to be of high interest despite of its challenging requirements. ${ }^{1,2}$ Due to the fact that these cyclobutane rings exist in many bioactive natural products (Figure 1-a), ${ }^{3-5}$ the need for such efficient, reliable, and benign synthesis methods is 
still under developing strategies to get a purely chiral strained carbocycle. Regardless the many different synthetic methodologies appeared in literatures to access cyclobutanes, ${ }^{6-11}$ the olefin dimerization via oxidative manners, which involves an active radical cation intermediate formation, represents a helpful and promising tactic to reach. The olefin dimerization was firstly reported by Ledwith ${ }^{12,13}$ and Bauld, ${ }^{14-16}$. In this regard, metal complexes ${ }^{17}$ and organic ${ }^{18,19}$ photoredox catalysis have been applied to promote such a nice cyclization. ${ }^{20-28}$ Recently, a major contribution to this field has been exploited by using catalytic amounts of HVIR ${ }^{29,30}$ in HFIP to investigate a stereoselective functionalization of alkenes. ${ }^{31-33}$ The HFIP has been shown to be a unique solvent due its significant role of hydrogen bonding ${ }^{34-36}$ that enables the HVIR to act as single electron oxidants. ${ }^{37-40}$ Based on the utility of the HVIR/HFIP, Donohoe and co-workers have developed a diastereoselective [2+2] cycloaddition of alkenes with remarkable results (Figure 1-b). ${ }^{41,42}$ The mechanism proposed involves a SEO of styrene 1 to a radical cation $\mathbf{1}^{+}$by HVIR followed by either HOMD, where dimerization proceeds with another molecule of styrene 1 in the presence of I(III) PIDA, or HETD, where dimerization proceeds with a different alkene 2 in the presence of I(V) DMP, to give the all trans cyclobutane product 3 after the re-addition of an electron to the product.

a) Bioactive natural products:
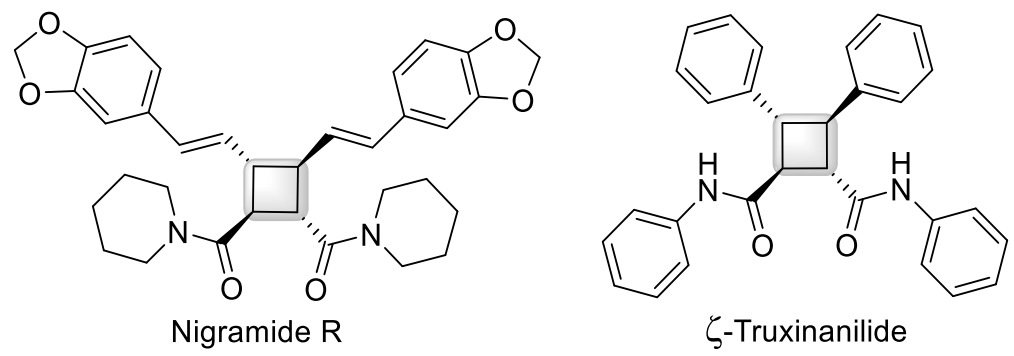

b) lodine-mediated dimerization of Styrenes:

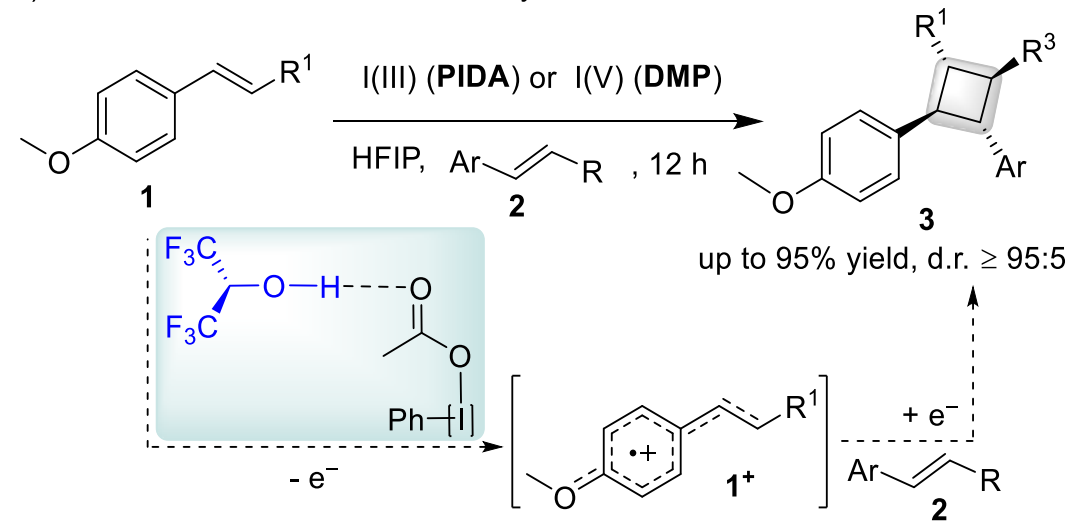

Figure 1. a) Examples of bioactive natural products containing cyclobutane ring. b) HOMD or HETD of styrenes with phenyliodine(III) diacetate (PIDA) or Dess-Martin periodinane (DMP), respectively .

The existence of hydrogen bonding interactions between the HFIP and PIDA has been proposed to be essential and the physical origin of the enhanced oxidative strength for the iodine reagent. ${ }^{43}$ In addition to the almost disappearance of $\mathrm{HO}$ signals from NMR experiments, the voltammetric peak potential experiments measured versus $\mathrm{Fc} / \mathrm{Fc}^{+}$demonstrated a shift in reduction potentials for PIDA $\left(E_{\mathrm{p}, \mathrm{c}}\right.$ in $\mathrm{ACN}=-1.32 \mathrm{~V}, E_{\mathrm{p}, \mathrm{c}}$ in $\mathrm{HFIP}=$ 
$-0.47 \mathrm{~V})$. The possibility of ligand exchange between HFIP and PIDA has been excluded and any altered reactivity to the oxidants is ruled out as the HFIP is a low nucleophilic solvent. ${ }^{43-47}$ All of the above-mentioned study concerns the first step of the reaction, the SEO step, and seems to us in need for further understandings, however, the subsequent steps that lead to the all trans cyclobutane ring are not considered, at least to the best of our knowledge, by other workers under these conditions. ${ }^{26,48-50}$ At this point, the reaction mechanism and reactivity of HVIR-mediate dimerization exclusively appears incomplete and warrants further attentions (Figure 2).. Therefore, we herein interpret DFT simulations on the HOMD and HETD that gives all trans cyclobutane under HVIRs with PIDA and DMP, respectively, featuring (1) the effect of HFIP on reactivity of this protocol, (2) the competition between the unwanted termination and propagations, (3) realizing the competition between homo- and heterodimerization via potential energy surfaces (PES) and quasiclassical trajectory molecular dynamics (QCTMD) simulations, (4) nature of cycloaddition, and (5) reactivities and stabilities of the correspondent charged radicals formed in this synthetic utility.

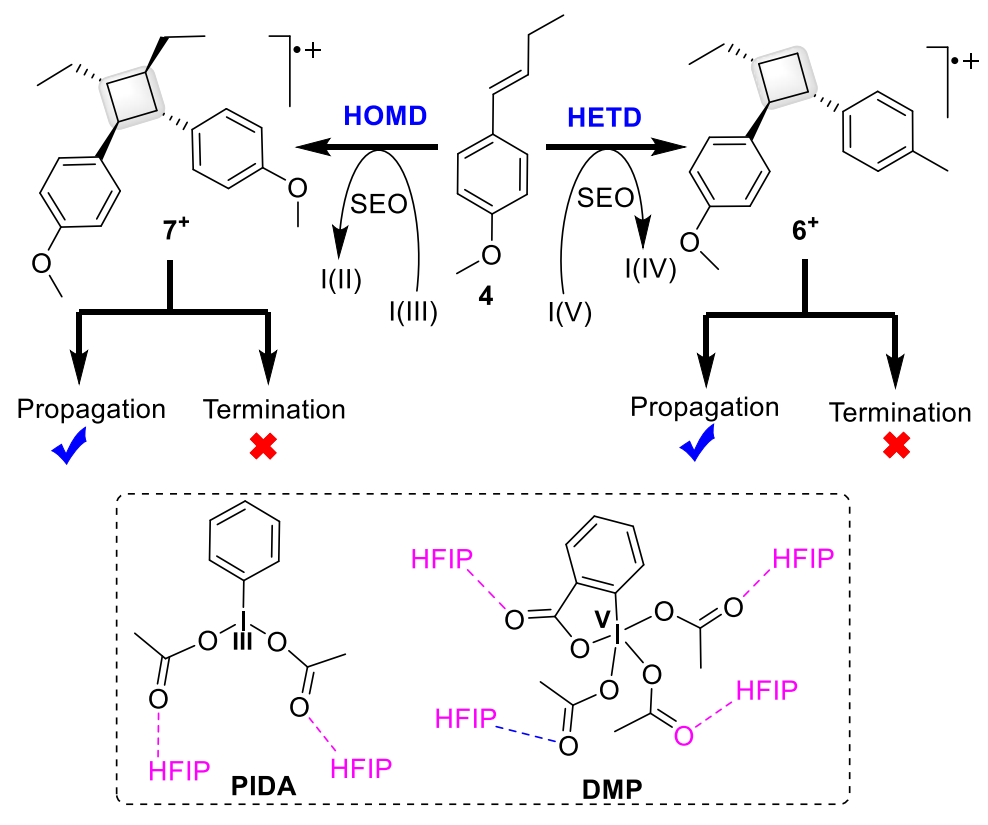

Figure 2. General representation of the HVIR [2+2] cycloaddition considered in this study, where iodine reagents are hydrogen bonded to HFIP explicitly.

\section{Results and Discussion}

To explore our HVIR-mediated dimerization of styrenes, we have divided our discussions into five distinct sections with the following order: validation of our strategy, mechanism of dimerization, competition between HOMD and HETD, and stability of the SEO and effect of substituents on SEO.

\section{Validation of strategy and level of theory}

The calculations were conducted in explicit and implicit HFIP. The explicit HFIP protocol means that every single acetate groups in PIDA and DMP is hydrogen bonded to one HFIP molecule to match the experimental 
conditions, whereas the implicit protocol is performed only with continuum solvation model based on density (IEFPCM-SMD). All structures were initially calculated using the $w \mathrm{~B} 97 \mathrm{XD} / 6-311+\mathrm{G}(\mathrm{d}, \mathrm{p}) / \mathrm{LANL} 2 \mathrm{DZ} / / 6-$ 31G(d)/LANL2DZ level of theory, however we found inconsistencies with the experimental results because of Fe and I atoms. Therefore, we carried out a basis set search on Fe and I atoms through running single point energy calculations on optimized structure by 6-31G(d)/LANL2DZ through comparison between measured and calculated voltammetric peak potentials for redox species of different substituted trans- $\beta$-methylstyrenes toward PIDA (Figure 10, see below). For Fc/Fc+, as shown in ESI, the cyclopentadienyl group in $\mathrm{Fc}$ was tested with a basis set of triple- $\zeta$ quality $(6-31 \mathrm{G}(\mathrm{d}, \mathrm{p}))$ to be consistent with the valence basis sets used for iron. We found that Def2-TZVPP/6-31G $(\mathrm{d}, \mathrm{p})$ level of theory gives the best agreement with the experimental redox potential values of different substituted trans- $\beta$-methylstyrenes using $\mathrm{Cp}_{2} \mathrm{Fe}$ (calculated $E^{1 / 2}=4.84 \mathrm{~V}$ ) as reference to calculate their redox potentials (see Figure 10). For iodine in PIDA, it was found that the basis set Def2-TZVPP for iodine and $6-311+\mathrm{G}(\mathrm{d}, \mathrm{p})$ for $\mathrm{C}, \mathrm{H}$, and $\mathrm{O}$ atoms gave the best agreement with experimental redox potentials (see ESI). Importantly, and under explicit protocol, the calculated value for PIDA $_{\text {HFIP }}+\mathrm{e}^{-} \rightarrow$ PIDA $^{-}$HFIP is $E^{1 / 2}$ $=4.25 \mathrm{~V}$ of peak potential for PIDA $\mathbf{H F I P}_{\mathrm{P}}$ is $E_{\mathrm{p}, \mathrm{c}}=-0.59 \mathrm{~V}$ versus calculated peak potential $\mathrm{Fc} / \mathrm{Fc}^{+}\left(E^{1 / 2}=4.84\right.$ $\mathrm{V})$ as a reference, leading to a good agreement with the measured peak potential for PIDA is $E_{\mathrm{p}, \mathrm{c}}=-0.47 \mathrm{~V}$. Under implicit protocol, the calculated value of non-hydrogen-bonded PIDA is $E^{1 / 2}=4.02 \mathrm{~V}$ of peak potential $E_{\mathrm{p}, \mathrm{c}}=-0.82 \mathrm{~V}$ versus $\mathrm{Fc} / \mathrm{Fc}^{+}$with a shifting to more negative value of $230 \mathrm{mV}$ less favorable than explicit

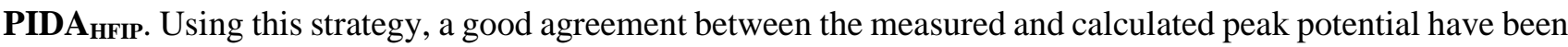
achieved as shown in Figure 10. Therefore, the $w$ B97XD/def2-TZVPP/6-311+G(d) level of theory is used for oxidants whereas the $w \mathrm{~B} 97 \mathrm{XD} / \mathrm{def} 2-\mathrm{TZVPP} / 6-31 \mathrm{G}(\mathrm{d}, \mathrm{p})$ level of theory is utilized for $\mathrm{Cp}_{2} \mathrm{Fe}$ in order to calculate the redox potentials.

\section{Mechanism of dimerization}

Our DFT investigations with the exploration of the HETD and HOMD facilitated by DMP and PIDA, respectively, are considered. The cyclobutane ring formation is investigated based on the SEO and single electron reduction (SER). When the SEO and SER are initiated and terminated, respectively, through only HVIR to get cyclobutane ring formed, this is a catalytic mechanism. The more plausible scenario is that the HVIR only initiates the reaction to get the styrene molecule radicalized by SEO and propagation of the reaction proceeds without HVIR and this is an initiated or propagated mechanism as the HVIR serves as an initiator.

To account better knowledge about the height barrier of SEO, free energy of activation for the SEO was calculated using four-point method proposed by Nelsen (see ESI). ${ }^{51-53} \mathrm{We}$ are convinced that this method result in a reasonable estimation of the electron transfer (ET) activation barrier.

It has been reported that HFIP plays a critical role with oxidizing agent rather than with the radical cation formed. ${ }^{43}$ The effect of explicit hydrogen bonding in our calculations is considered only on the SEO steps, namely the first and fourth reaction step (FRS and FoRS, respectively, as shown in Figure 3 and 4), whereas the cyclization steps, namely the second and third reaction steps (SRS and TRS, respectively, as shown in Figure 3 and 4), are proceeded with an implicit HFIP protocol. 
HETD pathway. The DFT results of HETD in the presence I(V) DMP with and without explicit HFIP molecules are shown in Figure 3. Generally, the explicit HFIP molecule decreases the overall energetic pathway for the HETD over the nonexplicit one due to the hydrogen bonding between DMP and HFIP which agrees with the findings of Donohoe and co-workers. The decrease is around $5 \mathrm{kcal} \mathrm{mol}^{-1}$ for the SEO step, FRS, and SER step, FoRS, and more than $6 \mathrm{kcal} \mathrm{mol}^{-1}$ for SRS and TRS. In the presence of explicit HFIP the free energy of activation for SEO for the FRS was found to be $20.2 \mathrm{kcal} \mathrm{mol}^{-1}$ to give radical cation $\mathbf{4}^{+}$and radical anion DMP $^{-}$HFIP as an endergonic step $\left(\Delta \mathrm{G}_{\mathrm{r}}=13.8 \mathrm{kcal} \mathrm{mol}^{-1}\right)$. In absence of explicit HIFP the barrier for SEO increased to $25.4 \mathrm{kcal} \mathrm{mol}^{-1}$ as a more endergonic process $\left(\Delta \mathrm{G}_{\mathrm{r}}=21.8 \mathrm{kcal} \mathrm{mol}^{-1}\right)$. An apparent increased in the $\mathrm{I}-\mathrm{O}$ bond distances, clearly represented for the perpendicular acetate units to the phenyl iodine. After the ET, the I-O bond distances elongate from 2.08 and $2.15 \AA$ to $2.63 \AA$ and $2.99 \AA$ when HFIP are not involved in calculations explicitly (see DMP and DMP- in Figure 3). Elongation is slightly less when HFIP is involved explicitly, where I-O bond length is $2.12 \AA$ is before the SEO and $2.55 \AA$ and $2.77 \AA$ are after the SEO (see $\mathbf{D M P}_{\text {HFIP }}$ and $\mathbf{D M P}^{-}{ }_{\text {HFIP }}$ in Figure 5-a). The increase in distance is reasonably attributed to the repulsions with the single unpaired electron existed on iodine (see spin density contours below).

All PES trails to find a concerted [2+2] cycloaddition TS for the cation cyclobutane formation $\mathbf{6}^{+}$are unsuccessful and, therefore, a two-step mechanism have been taken through the SRS and TRS. For the SRS, the head-to-head first $\mathrm{C}-\mathrm{C}$ bond formation was found to have a barrier of $8.3 \mathrm{kcal} \mathrm{mol}^{-1}$ via TS $\mathbf{8}^{+}$with bond length of $2.20 \AA$ along the TS is established, giving uncyclized intermediate $\mathbf{9}^{+}$with $\mathrm{C}-\mathrm{C}$ bond being formed at $1.58 \AA$ as a thermoneutral step of $0.1 \mathrm{kcal} \mathrm{mol}^{-1}$ (Figure 3). ${ }^{54}$ The TS $\mathbf{8}^{+}$shows a $\pi$ - $\pi$ stacking interaction of 3.6 $\AA$. A higher barrier TS of $13.1 \mathrm{kcal} \mathrm{mol}^{-1}$ was found without $\pi$ - $\pi$ stacking (see Figure S2 in ESI). It seems that the favorable $\pi-\pi$ stacking plays an important role in controlling the configurations of the product to be all trans cyclobutane. The presence of non-covalent interaction, $\pi-\pi$ stacking, for $\operatorname{TS} \mathbf{8}^{+}$and intermediate $\mathbf{9}^{+}$is shown by Reduced Density Gradient (RDG) analysis (Figure 5-c). ${ }^{55}$ Attractive $\pi-\pi$ interaction is clearly seen in the green areas between the two phenyl rings. The nature of interaction between $4^{+}$and 5 through TS $8^{+}$has a radical character due to SOMO-HOMO overlapping. The SOMO orbitals located on radical styrene $\mathbf{4}^{+}$is overlapped with the HOMOs of 5 with an energy gap if $4.03 \mathrm{eV}$ (see Figure S6). The radical cation intermediate $\mathbf{9}^{+}$cyclizes to the cationic cyclobutane $\mathbf{6}^{+}$, the TRS, in a low barrier step of $\Delta \mathrm{G}^{\ddagger}=7.1 \mathrm{kcal} \mathrm{mol}^{-1}$ with a long $\mathrm{C}-\mathrm{C}$ bond of $2.74 \AA$ along TS $\mathbf{1 0}^{+}$but in a slightly exergonic step $\left(\Delta \mathrm{G}_{\mathrm{r}}=-2.1 \mathrm{kcal} \mathrm{mol}^{-1}\right)$. Noticeably, the new $\mathrm{C}-\mathrm{C}$ bond formed in cyclobutane $6^{+}$is $1.64 \AA$ whereas all other $\mathrm{C}-\mathrm{C}$ bonds in the ring are $1.54 \AA$, and this is attributed to radical character as indicated by the partial delocalization shown by spin density and $\beta$-LUMO contours (see Figure S8).

To release the cyclobutane 6 , the radical cation $\mathbf{6}^{+}$undergoes SER by either the radical anion $\mathbf{D M P}^{-}{ }_{\text {HFIP }}$ or another styrene to propagate the reaction. The SER step to cyclobutane cation $\mathbf{6}^{+}$by DMP $^{-}$HFIP $^{-}$needs a barrier of $4.3 \mathrm{kcal} \mathrm{mol}^{-1}$ as an exergonic step $\left(\Delta \mathrm{G}_{\mathrm{r}}=-20.4 \mathrm{kcal} \mathrm{mol}^{-1}\right)$ (Figure 3). If this is true, the reaction would need longer time to complete as the reaction will need an initiation of higher energy step (FRS) in every single cyclobutane formation. Thus, propagation through oxidation of another styrene $\mathbf{4}$ by $\mathbf{6}^{+}$is more favored. The 
oxidation of styrene 4 by $\mathbf{6}^{+}$is nearly to be barrierless of $1.1 \mathrm{kcal} \mathrm{mol}{ }^{-1}$ as an exergonic step $\left(\Delta \mathrm{G}_{\mathrm{r}}=-6.6 \mathrm{kcal}\right.$ $\mathrm{mol}^{-1}$ ) (Figure 6).

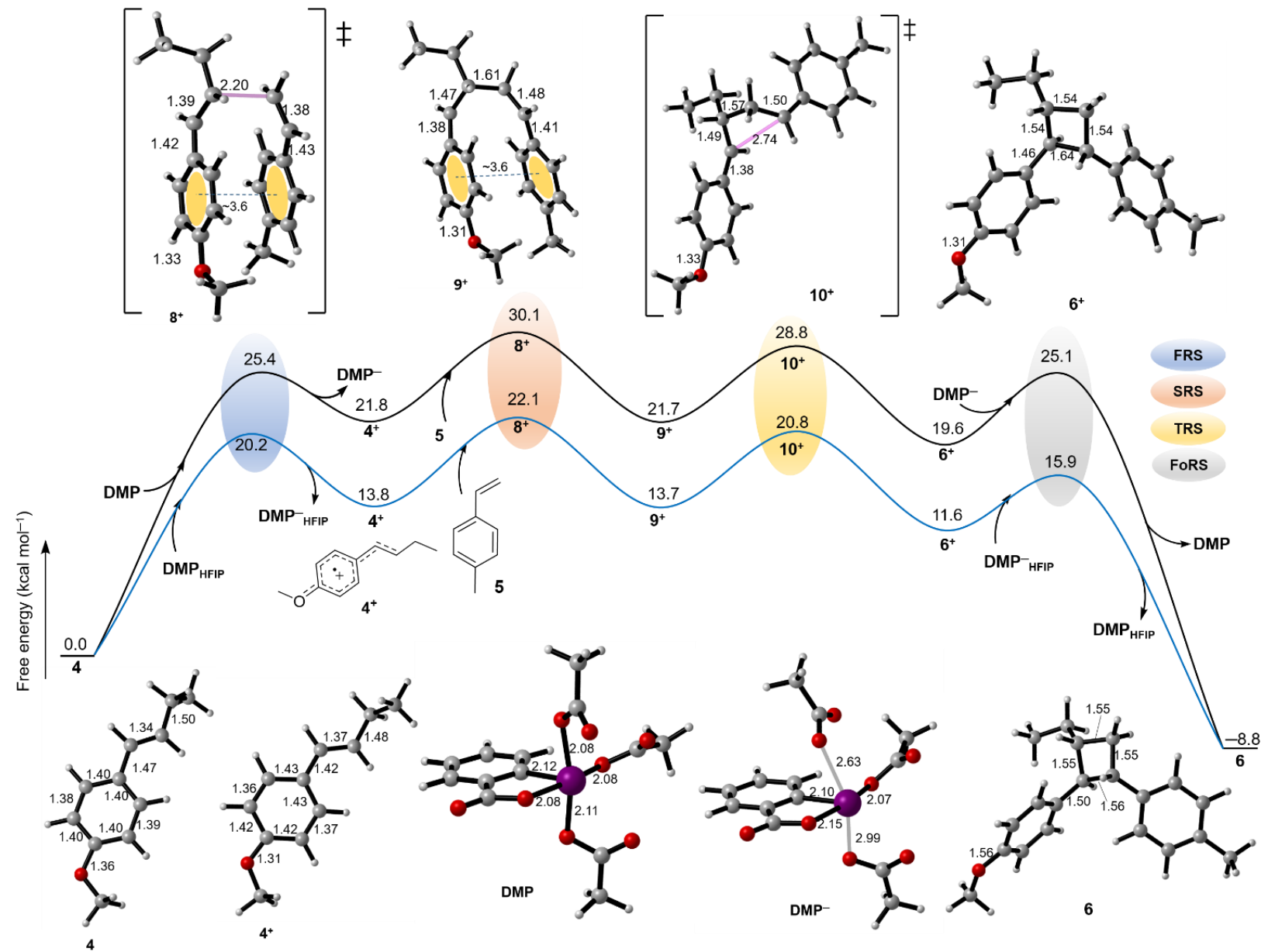

Figure 3. Free energy profile for the catalytic mechanism of DMP-mediated heterodimerization of styrenes (4) and (5), where SEO and SER proceed through only DMP to get cyclobutane ring formed, with explicit HFIP (blue pathway) and implicit HFIP (black pathway). Structures of $\mathbf{D M P} \mathbf{H F I P}_{\text {HF }}$ and $\mathbf{D M P}^{-}{ }_{\text {HFIP }}$ are shown in Figure 5-a. The FRS and FoRS are the first and fourth reaction step for the SEO and SER steps, respectively, and their barriers were estimated based on Nelsen four-point method explained in SI. The SRS and TRS are the second and third reaction steps showing the first and second $\mathrm{C}-\mathrm{C}$ bond formation.

HOMD pathway. Following the same strategy for HETD, the HOMD mechanism in the presence I(III) PIDA with and without explicit HFIP molecules is investigated and shown in Figure 4. As shown above with HETD, the explicit HFIP pathway shows lower energetic level than the nonexplicit pathway. The barrier of SEO, the FRS, was found to be $31.3 \mathrm{kcal} \mathrm{mol}^{-1}$ to give radical cation $\mathbf{4}^{+}$and anion PIDA $^{-}$HFIP as an endergonic step $\left(\Delta \mathrm{G}_{\mathrm{r}}\right.$ $=28.1 \mathrm{kcal} \mathrm{mol}^{-1}$ ). The SEO for HOMD is more endergonic than for the HETD. The calculated endergonicity

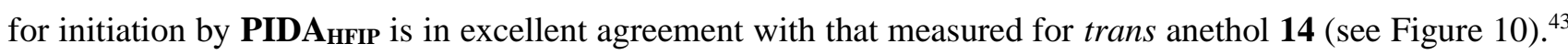
Following the SEO step the bond length of the acetate group to iodine, namely I-O bonds, increases from 2.15 $\AA$ to around $2.58 \AA$ for the non-hydrogen bonded PIDA (Figure 4) and to longer distances of $2.66 \AA$ and 2.78 $\AA$ for the hydrogen-bonded one PIDAHFIP (Figure 5-b) for the reason mentioned above for SEO by DMP $\mathbf{P H I P}_{\text {. }}$ 


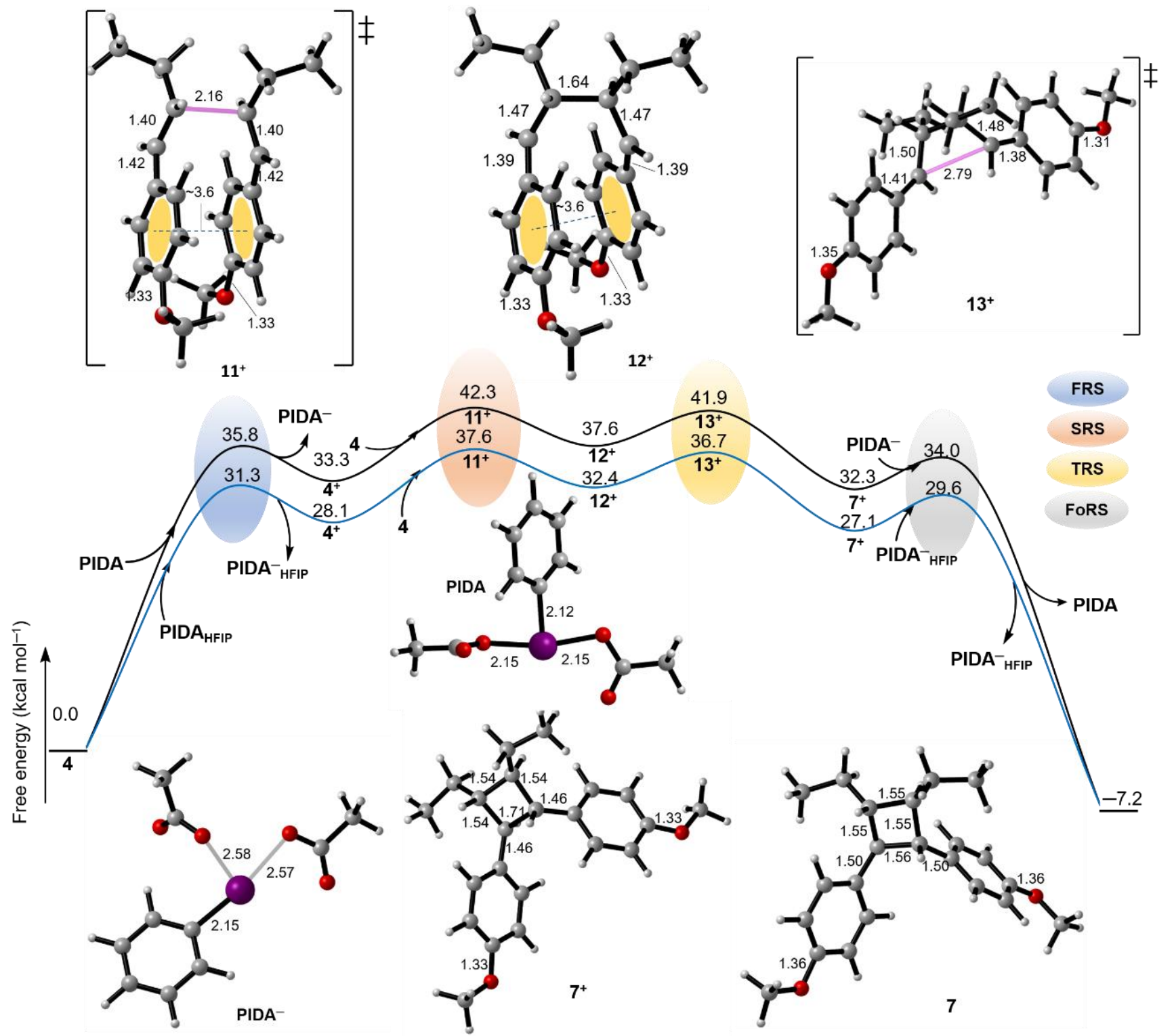

Figure 4. Free energy profile for the catalytic mechanism of PIDA-mediated homodimerization of styrenes (4), where SEO and SER proceed through only PIDA to get cyclobutane ring formed, with explicit HFIP (blue pathway) and implicit HFIP (black pathway). Structures of PIDA HFIP and PIDA ${ }^{-}$HFIP are shown in Figure 5-b. The FRS and FoRS are the first and fourth reaction step for the SEO and SER steps, respectively, and their barriers were estimated based on Nelsen four-point method explained in ESI. The SRS and TRS are the second and third reaction steps showing the first and second $\mathrm{C}-\mathrm{C}$ bond formation.

The process for $\mathbf{4}^{+} \rightarrow \mathbf{1 2}^{+}$, the SRS, has a reasonable barrier of $9.5 \mathrm{kcal} \mathrm{mol}^{-1}$ via $\pi-\pi$ stacked head-to-head TS $\mathbf{1 1}^{+}$with bond length of $2.16 \AA$ to give the cationic uncyclized intermediate $\mathbf{1 2}^{+}$as an endergonic step of 4.3 $\mathrm{kcal} \mathrm{mol}^{-1} .{ }^{54}$ The favorable non-covalent interaction that represents the $\pi$ - $\pi$ stacking interaction between the two phenyl rings is shown in Figure 5-d. A higher barrier TS of $12.9 \mathrm{kcal} \mathrm{mol}^{-1}$ was found for the first C-C bond formation when aromatic rings are not stacked (Figure S2). Likely to TS $\mathbf{8}^{+}, \mathrm{TS} \mathbf{1 1}^{+}$has a radical character with an energy gap of $4.86 \mathrm{eV}$ (see Figure S7) which is higher than for the HETD $\left(\mathbf{4}^{+}\right.$and $\left.\mathbf{5}\right)$. The first $\mathrm{C}-\mathrm{C}$ bond 
formation in $\mathbf{1 2}^{+}$is longer than for that found for the uncyclized heterodimerized intermediate $\mathbf{9}^{+}$. The cyclization, TRS, is a low barrier step of $4.3 \mathrm{kcal} \mathrm{mol}^{-1}$ through TS $\mathbf{1 3}^{+}$with $\mathrm{C}-\mathrm{C}$ bond at $2.16 \AA$ is seen to give the cationic homodimerized cyclobutane $7^{+}$as an exergonic step $\left(\mathbf{1 2}^{+} \rightarrow \mathbf{7}^{+}, \Delta \mathrm{G}_{\mathrm{r}}=-5.3 \mathrm{kcal} \mathrm{mol}{ }^{-1}\right)$. Upon formation of $\mathbf{7}^{+}$, the unpaired electron has totally delocalized over the entire system of $\mathbf{7}^{+}$(see Figure S8) and resulted in an increase in the new $\mathrm{C}-\mathrm{C}$ bond to be $1.71 \AA$, being longer than for $\mathbf{6}^{+}$.
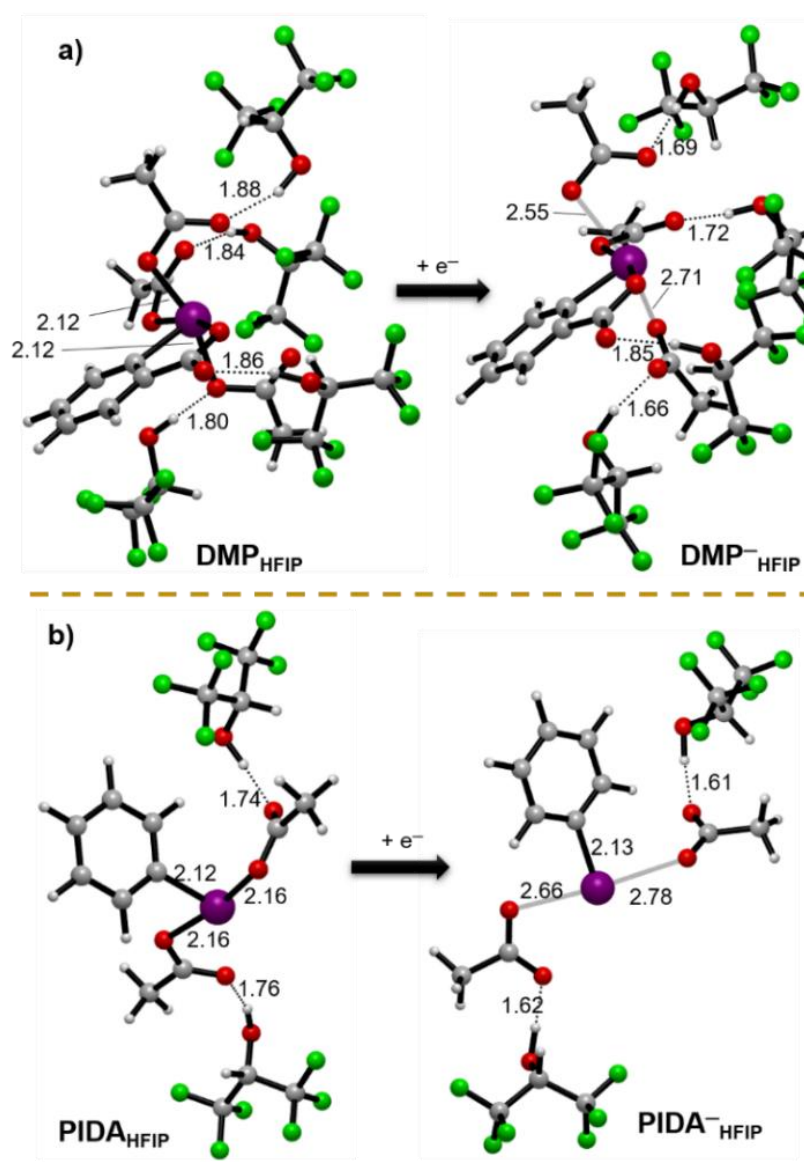

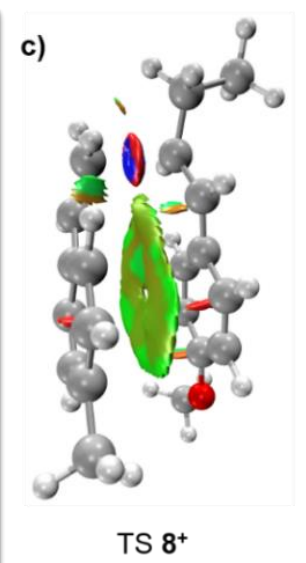

TS $8^{+}$

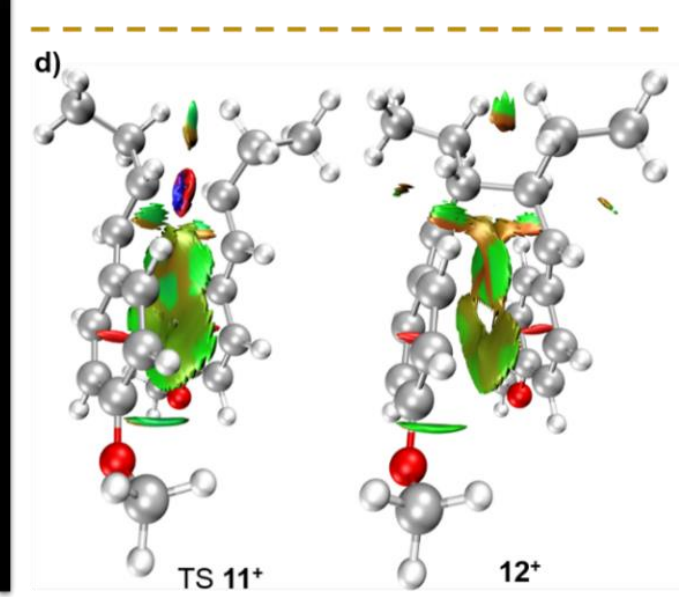

Figure 5. (a) and (b)Optimized hydrogen-bonded neutral and anion species of DMP HFIP $_{\text {and PIDA }}$ HFIP. (c) and (d) Reduced Density Gradient (RDG) isosurface (isovalue at 0.5 ) for TSs $\mathbf{8}^{+}$and $\mathbf{1 1}^{+}$and intermediates $\mathbf{9}^{+}$and $\mathbf{1 2}^{+}$, where attractive $\pi-\pi$ stacking interactions are shown in green area, whereas red and blue area indicates steric repulsion and strong interactions, respectively.

The release of neutral homodimerized cyclobutane 7 via propagation process (Figure 6) is calculated to be favored over SER by radical anion PIDA- ${ }_{\text {HFIP }}$ (Figure 4). The oxidation of styrene 4 by $\mathbf{7}^{+}$is found to be nearly barrierless of $1.5 \mathrm{kcal} \mathrm{mol}^{-1}$ as an exergonic step $\left(\Delta \mathrm{G}_{\mathrm{r}}=-6.3 \mathrm{kcal} \mathrm{mol}^{-1}\right)$ (Figure 6). The SER by PIDA- ${ }_{\text {HFIP }}$ requires higher barrier of $2.5 \mathrm{kcal} \mathrm{mol}^{-1}$ as a result of the high exergonicity $\left(\Delta \mathrm{G}_{\mathrm{r}}=-34.4 \mathrm{kcal} \mathrm{mol}^{-1}\right)$ needed to release the neutral cyclobutane 7 (Figure 4). This would indicate that reorganization energy plays an important role in driving the reaction toward propagation over termination by radical anionic HVIR. Overall, this would mean that the reaction is only initiated by the DMP or PIDA reagents and a very low concentration of radical cation $\mathbf{4}^{+}$might be enough to reach the HETD or HOMD completed. 
a) HETD

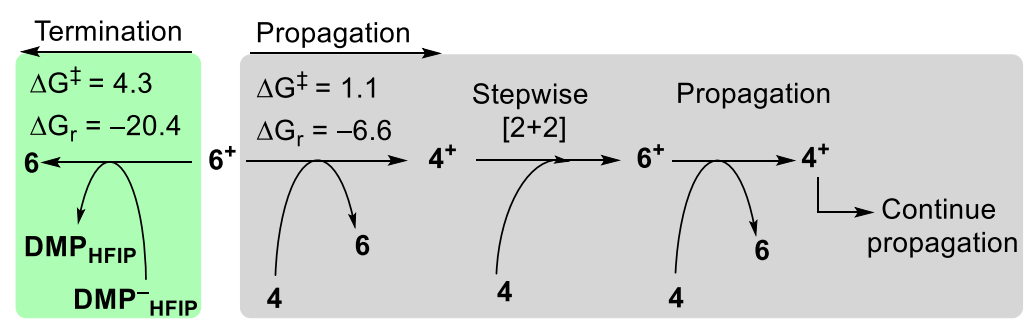

b) HOMD

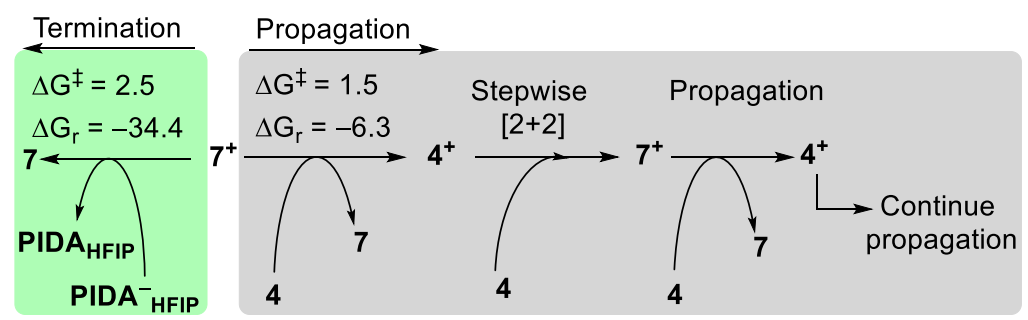

Figure 6. Propagation of HOMD and HETD.

\section{Competition between HETD and HOMD}

In general, the initiative SEO step from styrene $\mathbf{4}$ is shown to be more reactive with DMP catalyst since the SEO occurs with the more deficient catalyst I(V) $(\mathrm{LUMO}=-1.56 \mathrm{eV})$ over less deficient one I(III) (LUMO = $-0.73 \mathrm{eV}$ ), apparently indicating that the initiation in HETD is faster than HOMD under DMP conditions. However, and from a synthetically perspective point of view, there is a competition between both processes which is experimentally seen. This concerns the fact that HOMD can relatively be accessed via HETD conditions. Our simulations have recognized these complications and shown to be problematic after initiation, FRS, takes place via I(V) catalyst (DMP $\left.\mathbf{P F I P}_{\mathbf{H F}}\right)$. The calculations indicate that once the radical cation $\mathbf{4}^{+}$is formed, entering homo [2+2] cycloaddition is competitive. The results above (Figure 3 and 4) reveal that the HOMD starts with a barrier of $9.5 \mathrm{kcal} \mathrm{mol}^{-1}$ via TS $\mathbf{1 1}^{+}$whereas HETD starts with lower barriers of $8.3 \mathrm{kcal} \mathrm{mol}^{-1}$ via TS $\mathbf{8}^{+}$, implying difference between HOMD and HETD to be $\Delta \Delta \mathrm{G}^{\star}=1.2 \mathrm{kcal} \mathrm{mol}^{-1}$. This is in general good agreement with the experimental findings, in which the HETD reaction proceeds an equivalent ratio of styrenes 5 to 4 of $2: 1 .{ }^{41}$ A further evidence for the competition between HOMD and HETD is emerged form QCT molecular dynamics of the first $\mathrm{C}-\mathrm{C}$ bond formation (shown below).

Quasiclassical trajectory molecular dynamics (QCTMD) calculations. QCTMD simulations were utilized to understand the chronological character for formation of first $\mathrm{C}-\mathrm{C}$ bonds in order to demonstrate the competition between HETD and HOMD cycloadditions (Figure 7). ${ }^{56-62} 44$ and 63 trajectories were generated starting from the TSs $\mathbf{8}^{+}$and $\mathbf{1 1}^{+}$, respectively, in which forward and backward propagations $(\mathrm{t}=0 \mathrm{fs})$ are initiated showing the typical reactive bonds toward either cationic uncyclized intermediates $\left(\mathbf{9}^{+}\right.$and $\left.\mathbf{1 2}^{+}\right)$or reactants (styrene $\mathbf{4}^{+}, \mathbf{4}$ and 5). No recrossing is observed in our simulation. The C3-C4 distance is rapidly shortened to $\sim 1.6 \AA$ in most trajectories, and the bond remains in the whole trajectory once formed although for 
a small proportion of trajectories the $\mathrm{C} 3-\mathrm{C} 4$ distance oscillates in the range between $1.6 \AA$ and $2.0 \AA$. By recording the timing for the $\mathrm{C} 3-\mathrm{C} 4$ distance to be shortened below $1.6 \AA$, we obtained the average timing for the first $\mathrm{C}-\mathrm{C}$ bond formation at 43.0 and $47.0 \mathrm{fs}$ for $\mathrm{HETD}^{+}$and $\mathrm{HOMD} \mathbf{1 1}^{+}$, respectively. It is interesting that although the average timing is similar, there are more trajectories exhibiting larger timing for $\mathrm{C}-\mathrm{C}$ bond formation for the HOMD pathway, which may indicate a flatter potential energy surface in the post-transition state period. Comparison of the average time for the first $\mathrm{C}-\mathrm{C}$ bond formation through $\mathrm{TS} \mathbf{8}^{+}$and $\mathrm{TS} \mathbf{1 1}^{+}$reveals a short timing gap of $4.0 \mathrm{fs}$. Also, the timing for first $\mathrm{C}-\mathrm{C}$ bond formation for the unstacked TSs were obtained and shown a short timing gap where 46 fs for HOMD, derived from 24 trajectories, and 44 fs for HETD, derived from 20 trajectories (see Figure S3 in ESI). Overall, the very small timing gap between both pathways is in good consistence with the coexistence of HETD and HOMD pathways and explain why both pathways are competitive.
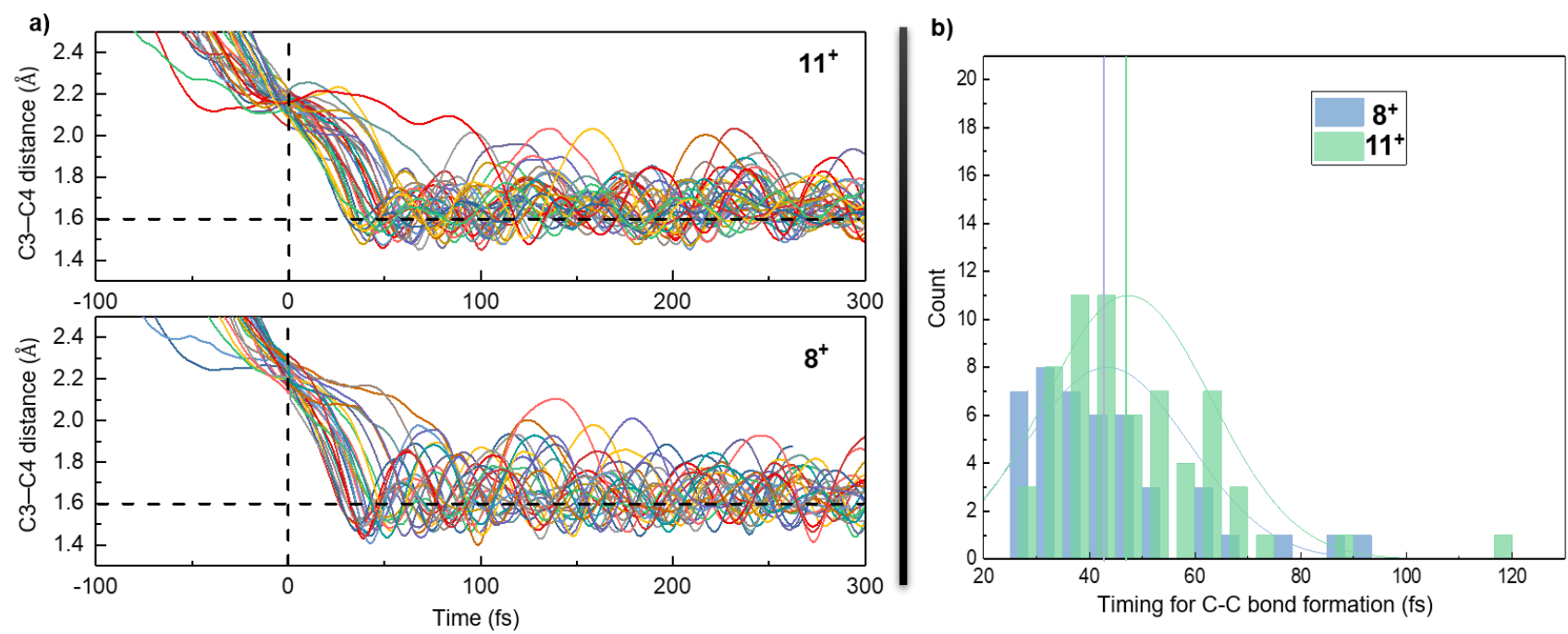

Figure 7. (a) Evolution of the $\mathrm{C}-\mathrm{C}$ distance corresponding to the first $\mathrm{C}-\mathrm{C}$ bond formation along quasiclassical trajectories initialized from TSs $\mathbf{8}^{+}$and $\mathbf{1 1}^{+}$calculated by the $w \mathrm{~B} 97 \mathrm{XD} / 6-31 \mathrm{G}(\mathrm{d})$ level of theory. All trajectories start from the initial geometry ( $\mathrm{t}=0 \mathrm{fs})$ generated by adding a random displacement to the transition state, and both directions are shown in positive and negative part of the horizontal axis. (b) A histogram for the $\mathrm{C}-\mathrm{C}$ bond formation timing, where the average timing for each reaction is shown by the vertical line.

\section{Stability of the SEO step}

The stability of the radical cations and anions formed from the SEO step can be rationalized at least qualitatively on the basis of frontier molecular orbital analysis. Simply, removing an electron from styrene 4, represented as highest occupied molecular orbitals (HOMO), to the HVIR, represented as lowest unoccupied molecular orbitals (LUMO), generates the radical anion and cation. These charged species are generally stabilized by the frontier orbitals of the charged radicals, where HOMOs for anions and LUMOs for cation. 
FMO of radical cation $\mathbf{4}^{+}$. The HOMOs on styrene $\mathbf{4}$ are a combination of two degenerate HOMOs (HOMO and HOMO-1) came from the double bond and para-phenyl ring (Figure 8-a). The SEO leads to stabilizations for cations to be achieved from HOMOs and LUMOs. As shown in Figure 8-a, removal of an electron from HOMO of styrene 4 splits its HOMO into $\beta$-LUMO and $\alpha$-SOMO (singly occupied MO). The removal of electron from the HOMO lessens the HOMO energy to more than half because the orbital is singly- rather than doubly-occupied. Secondly, a stabilization in the LUMO of radical cation $\mathbf{4}^{+}$raises to more than double after electron removal. Thirdly, the remaining two electrons in the HOMO-1 have bonding interactions and a preference for radical cation to adopt interaction is existed. At this point, the radical cation gains stabilizations when the HOMO is no longer doubly occupied because the unpaired electron is delocalized by which a prominent $\pi$ electron delocalization distributed from the styrene double bond to the phenyl ring. The spin density, $\rho_{(\alpha)}-\rho_{(\beta)}$, provides information about the localization of the unpaired electron. While the calculated spin density is majorly accounted from the HOMOs (HOMO energy is $\alpha=-12.15 \mathrm{eV}$ and $\beta=-6.79 \mathrm{eV}$ ), the contours of the spin density clearly display an entire delocalization over the system (see blue box in Figure 8a) although the contour for spin density is similar to the SOMO.

Under HFIP SEO conditions, the oxidants DMP HFIP $_{\text {and }}$ PIDA electron addition, majorly attributed to the hydrogen bonding interactions that substantially lower the energy of the LUMOs and at some level the HOMOs. Therefore, we will discuss these stabilities as before and after electron addition.

FMO of radical anions DMP ${ }^{-}{ }_{\text {HFIP }}$ and PIDA $^{-}{ }_{\text {HFIP. }}$ The effect of electron addition on splitting of LUMOs for HVIRs with and without hydrogen bonding is shown in Figure 8-b,c. Further visualizations of FMOs for DMP ${ }^{-}$ and PIDA $^{-}$are provided in ESI. In the case of DMP $_{\text {HFIP }}$, the electron is added to the LUMO leading to split its orbitals into $\alpha$-SOMO and $\beta$-LUMO. This lessens the raise in $\alpha$-SOMO energy to more than triple times of the

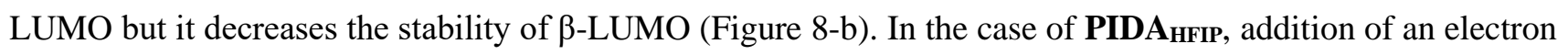
makes the energy of SOMO stabilized by more than seven times of the LUMO with the $\beta$-LUMO being slightly stabilized too (Figure 8-c). The unpaired electron is localized on the iodine center, perpendicularly to the phenyl ring as indicated by the orbital contours of SOMO and spin density for $\mathbf{D M P}^{-}{ }_{\text {HFIP }}$ and PIDA $^{-}{ }_{\text {HFIP. Noticeably, }}$ we have seen that initiation by DMP $_{\text {HFIP }}$ is more thermodynamically favorable than by PIDA $\mathbf{A F I P}_{\text {HF }}$ A reasonable explanation would be more likely emerged from the fact that the SOMO energy of the $\mathbf{D M P}^{-}{ }_{\text {HFIP }}$ is lower than

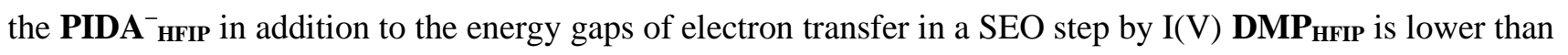

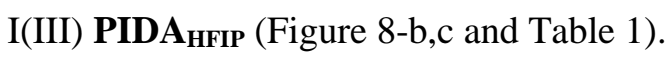


(a)

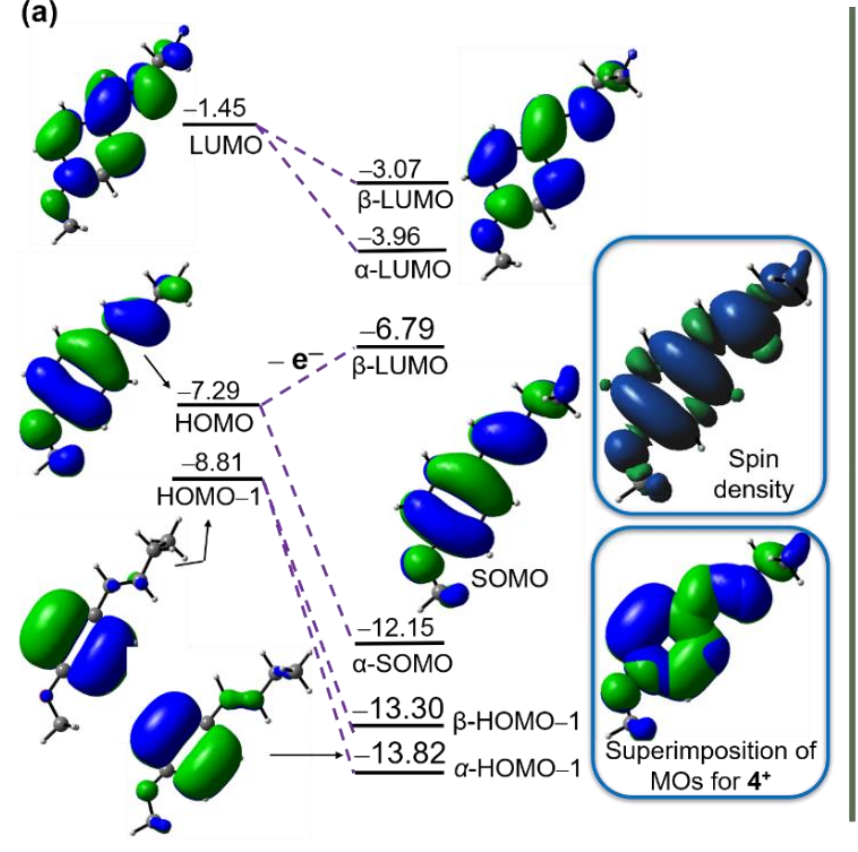

(b)
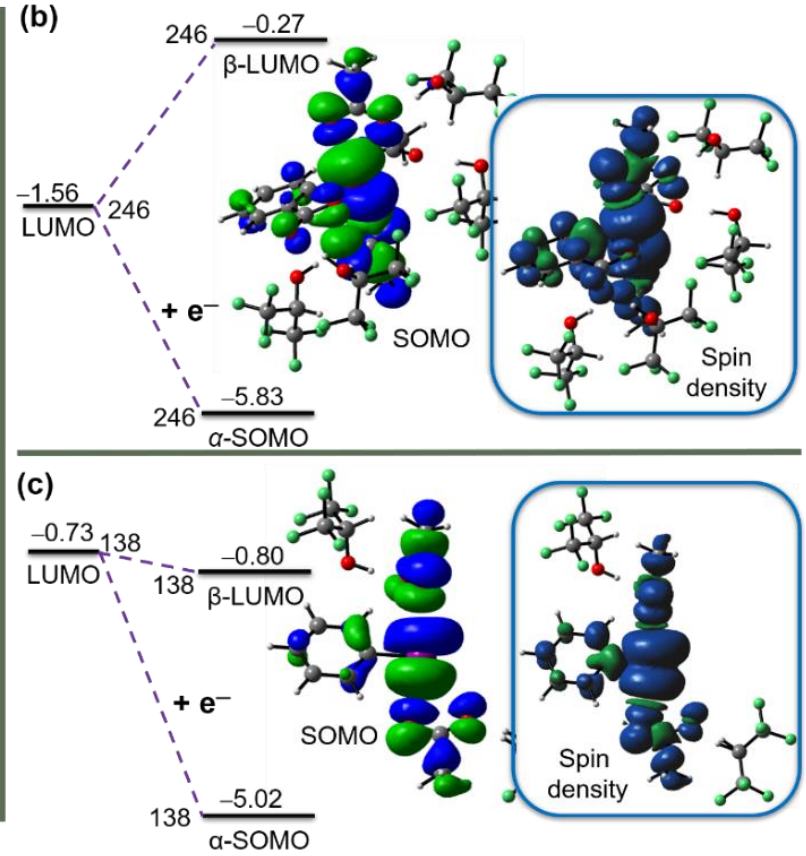

Figure 8. (a) Involvement of the FMOs for 4 before (left) and after (right) electron removal. The removal of an electron splits the HOMO into $\beta$-LUMO and $\alpha$-SOMO that have identical wave functions. (b) Splitting of LUMO into $\alpha$-SOMO and $\beta$-LUMO after electron addition to DMP $\mathbf{P F I P}_{\text {(a) }}$ and PIDA $_{\text {HFIP }}$ (b). Spin density of the correspondent radical cations and anions are in blue box.

FMO of neutral DMP HFIP $_{\text {and PIDA }}$ HFIP. The effect of hydrogen bonding interactions on stability of the HOMOs and LUMOs accounted for neutral HVIRs before electron addition is presented in Figure 9. Two important information can be seen from Figure 9. First, the hydrogen bonding generally lessens the energy of LUMOs and HOMOs of DMP and PIDA. The impact of lowering the energetic levels of LUMOs is seen to be greater on DMP HFIP $_{\text {than }}$ PIDA $_{\text {HFIP. The LUMO energy of DMP }}$ HFIP decreases by around $1.04 \mathrm{eV}(-0.52 \rightarrow$ $-1.56 \mathrm{eV}$, Figure 9-a) whereas a lower decrease of $0.82 \mathrm{eV}(+0.09 \rightarrow-73 \mathrm{eV}$, Figure 9-b) is obtained for

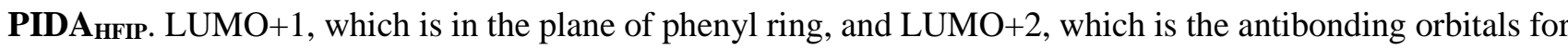
the phenyl ring, are stabilized by $1.01 \mathrm{eV}(-0.27 \rightarrow-1.28 \mathrm{eV})$ and $0.81 \mathrm{eV}(+0.36 \rightarrow-0.45 \mathrm{eV})$ for DMP, respectively. For PIDA, LUMO+1 and LUMO+2 are stabilized by $0.63 \mathrm{eV}(+0.68 \rightarrow+0.05 \mathrm{eV})$ and $0.38(+1.28$ $\rightarrow+0.90 \mathrm{eV}$ ), respectively. This indicates that hydrogen bonding makes the iodine more deficient by lowering the energy of antibonding orbitals.

Second, stabilization of the HOMOs is also seen but the effect on DMP is different. The HOMO orbitals on PIDA have similar contours before and after PIDA being hydrogen bonded. The HOMO and HOMO-1 that were belong to the axial and equatorial (to the plane of phenyl ring) antibonding orbitals on DMP become located on HFIP molecules, specifically on the OH of HFIP, although HFIP is low nucleophilic solvent. This would indicate that the effect of nucleophilicity of acetate groups on iodine decreases substantially when they are hydrogen bonded. This case is not seen in I(III) PIDA because DMP is already higher oxidation state in which hydrogen bonding makes I(V) more deficient and reactive reagent. Overall, HIFP lowers the HOMOs of 
acetate groups and, subsequently, the LUMO of the iodine center decreases to a level that makes it a capable species for SEO.

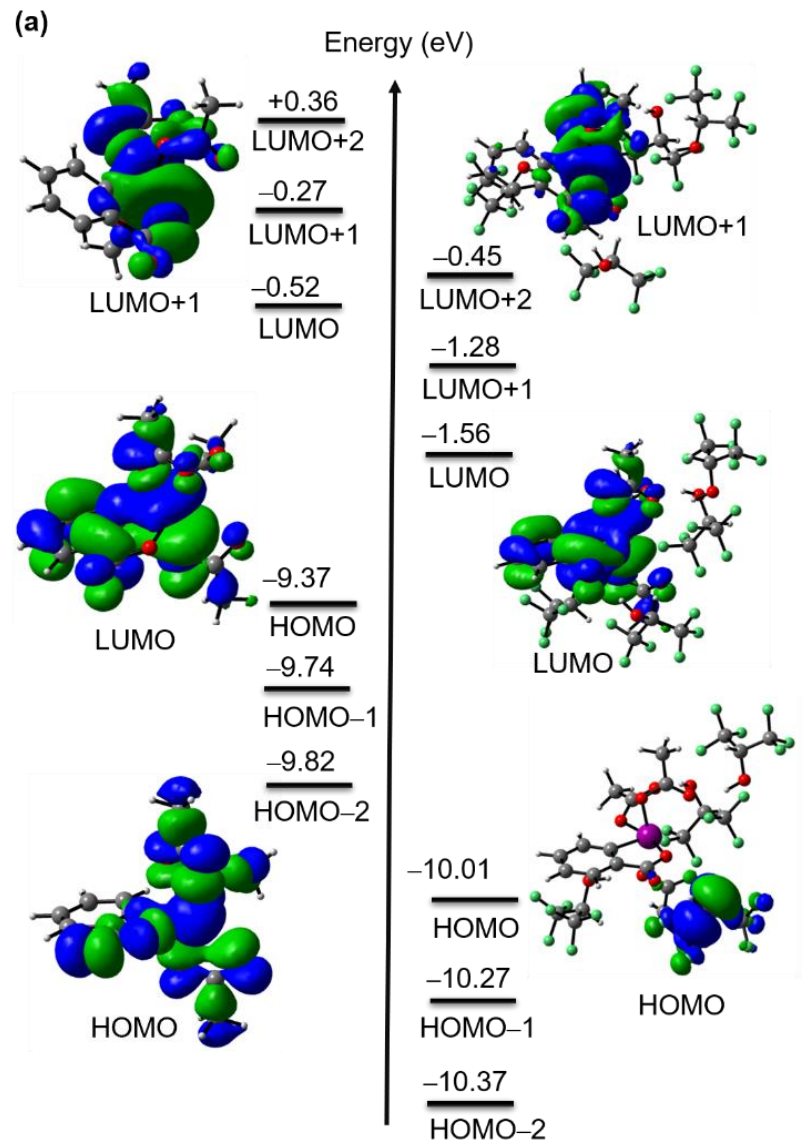

(b)

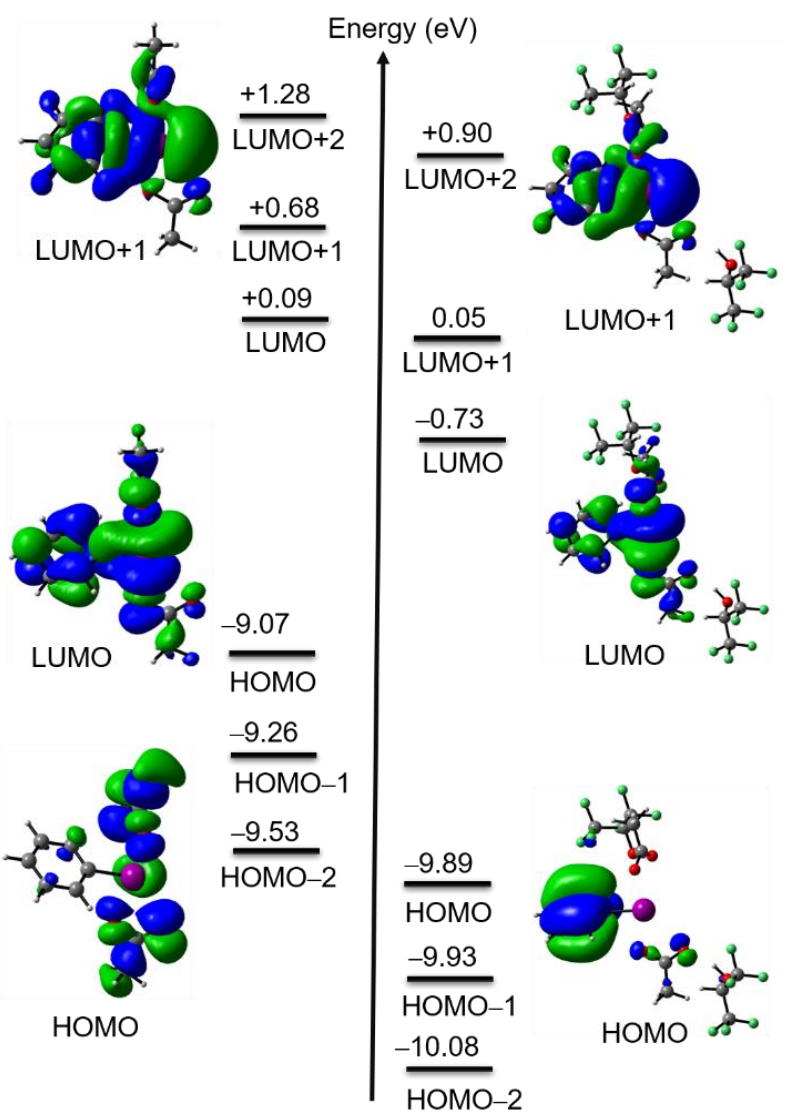

Figure 9. FMOs of the DMP (a) and PIDA (b) before electron addition, showing the effect of hydrogen bonding interactions on stabilities of the HOMOs and LUMOs of each reagent. The contours of the other orbitals are shown in SI.

\section{Effect of substituents on SEO}

Based on the above findings, we have investigated the effect of substituents on the SEO to gain a broader vision that makes a styrene successfully active. First, comparison between calculated and measured redox potentials of different substituted trans- $\beta$-methylstyrenes $\mathbf{1 4 - 2 4}$ is indicated in Figure 10. Our strategic DFT simulations present a very good agreement with the experimental redox potentials and free energy of reoxidation accordingly. The explicit-involved HFIP calculations are consistent with experimental results than inexplicit calculations (for comparison see ESI). The calculated results appeared in Figure 10-b indicate is a deviation from experimental values of around $0.12 \mathrm{eV}$ which is in agreement with the mean absolute error in ionization energy $\left(2.74 \mathrm{kcal} \mathrm{mol}^{-1}\right)$ reported for $w \mathrm{~B} 97 \mathrm{XD} .{ }^{63}$ Notably, the measured and calculated potentials refer to the important role of a $p$-methoxy group in the success of a styrene toward dimerization, however, the absence of $p$-methoxy motif resulted in unsuccessful dimerization with PIDA/HFIP. ${ }^{43}$ Using voltammetric oxidation peak potentials to understand the reactivity is relatively evident. Styrenes with high voltammetric oxidation potentials 
failed to undergo [2+2] cycloaddition under PIDA/HFIP conditions due to the initiation (SEO, FRS) become comparatively thermodynamically disfavorable, especially for more electron deficient styrenes. Styrenes $\mathbf{1 4}$ 19 were synthetically reactive and $\mathbf{2 0}-\mathbf{2 4}$ were unreactive. ${ }^{43}$ Broadly saying, and as indicated by Donohoe and co-workers it seems that the styrenes that exhibit oxidative peak potentials $\left(E_{\mathrm{p}, \mathrm{a}}\right)$ below $\sim+1.0 \mathrm{~V} \mathrm{vs} \mathrm{Fc} / \mathrm{Fc}^{+}$in HFIP lead to [2+2] cycloaddition product although the correlation seems relatively not applied on the productive styrenes 18 and 19 and the unproductive styrenes 20 and 21 when subject to PIDA/HFIP conditions. Further

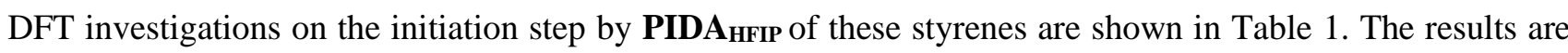
compared with those predicted by $\mathbf{D M P}_{\text {HFIP }}$ (Table 1). Experimentally, the styrenes $\mathbf{1 4}-\mathbf{2 4}$ were tested only

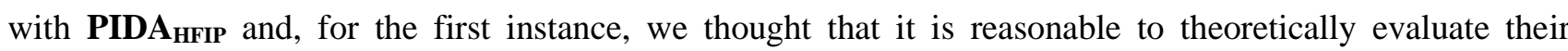
reactivities toward higher oxidation state of HVIR. We will first explain the results of SEO by PIDA HFIP. $_{\text {. }}$

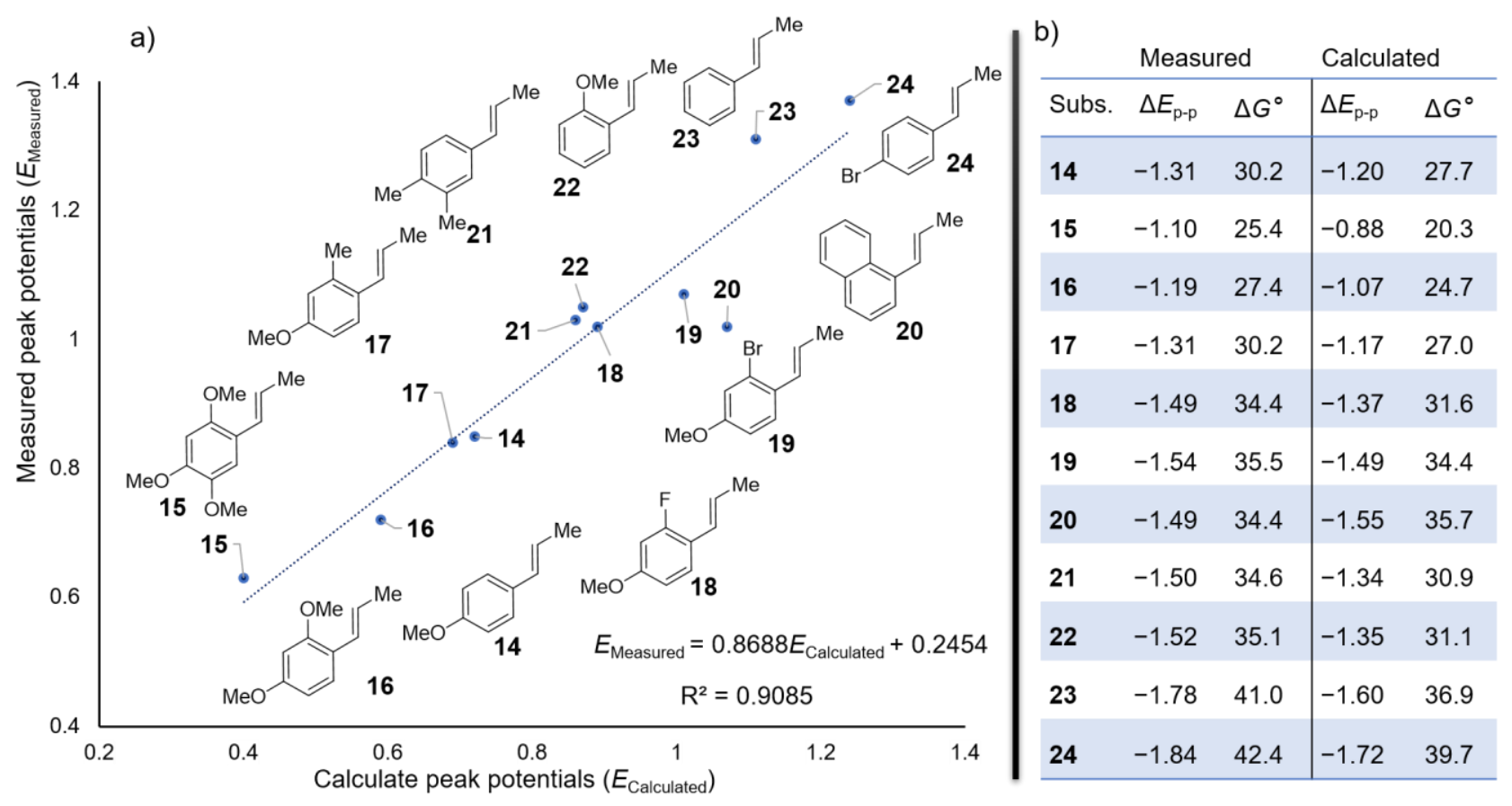

Figure 10. (a) Agreement between measured and calculated voltammetric peak potentials (in V) for redox species of different substituted trans- $\beta$-methylstyrenes $\mathbf{1 4}$ - 24. (b) Differences in the reduction and oxidation peak potentials (in $\mathrm{V}$ ) and their Gibbs free energies (in $\mathrm{kcal} \mathrm{mol}^{-1}$ ) for styrenes $\mathbf{1 4}-\mathbf{2 4}$. Styrenes $\mathbf{2 0}-\mathbf{2 4}$ did not undergo PIDA [2+2] cycloaddition. The measured results were obtained versus $\mathrm{Fc} / \mathrm{Fc}^{+}$, as measured at 100 $\mathrm{mV} \mathrm{s}^{-1}$ taken from ref 43 . The calculated $\mathrm{Fc} / \mathrm{Fc}^{+}$is $E^{1 / 2}=4.84 \mathrm{~V}$ in HFIP. The calculated value for PIDA $\mathbf{H F I P}_{+}$ $\mathrm{e}^{-} \rightarrow$ PIDA $^{-}$HFIP is $E^{1 / 2}=4.25 \mathrm{~V}$. Measured peak potential for PIDA is $E_{\mathrm{p}, \mathrm{c}}=-0.47 \mathrm{~V} .{ }^{43}$ Th calculated peak potential for PIDAHFIP $E_{\mathrm{p}, \mathrm{c}}=-0.59 \mathrm{~V}$.

The DFT calculations on MOs analysis clearly evidence that from the top to the down of the table the more electron-rich styrene system the highest HOMOs and the more reactive toward the SEO, i. e. HOMOs decrease from the top to the down of the table. Following an electron removal from styrenes, the stability of the degenerated orbitals, $\alpha$-SOMO and $\beta$-LUMO, increase with the decrease in the HOMOs. In this regard, the calculated energy gaps for an electron transfer to PIDA $\mathbf{H F I P}\left(\mathrm{E}_{\mathrm{g}}{ }^{\mathrm{c}}=\mathrm{LUMO}\left[\mathbf{P I D A} \mathbf{H F I P}_{\mathbf{H}}\right]-\mathrm{HOMO}[\mathrm{styrene}]\right)$ 
increase from the top to the down of the table with the highest one reported for the styrene $\mathbf{2 4}$ (Table 1). The energy barriers of initiation for substrates $\mathbf{1 4}-\mathbf{2 4}$ indicated in Table 1 have a correlation with experimental $E_{\mathrm{p}, \mathrm{a}}$ peak potentials and Gibbs free energy of SEO step. Those substrates with low experimental $E_{\mathrm{p}, \mathrm{a}}$ have low $\Delta \mathrm{G}_{\mathrm{r}}$ and correspondingly lower $\Delta \mathrm{G}^{\star}$ and vice versa. The SEO step that leads to a more thermodynamically favorable radical cation formation makes the cycloaddition more successful, but this is more likely dependent on the kinetics of this step that requires Gibbs free energy $\left(\Delta \mathrm{G}_{\mathrm{r}}\right)$ and reorganization energy $(\lambda)$ based on Marcus theory. However, it turns out that the calculated barriers for all styrenes indicate that the thermodynamic term represents a major contribution in the initiative barrier, and the reorganization term evidently becomes less effective. In agreement with the experimental findings, the unreactive styrenes $\mathbf{2 0}-\mathbf{2 4}$ were found to undergo highly initiative barriers whereas reactive styrenes $14-19$ are generally require lower energy to initiate the reaction. Furthermore, a good correlation between the calculated FMO energies and free energies of activation and reaction is relatively apparent. The removal of an electron from a low HOMO styrene gives a low $\alpha$-SOMO and $\beta$-LUMO radical cation and this, consequently, leads to a lower energy gap and free energy of activation.

Table 1. Calculated energies for the SEO step by I(III) PIDA HFIP $_{\text {and }}$ DMP $_{\text {HFIP }}$ of different substituted trans$\beta$-methylstyrenes $\mathbf{1 4}-\mathbf{2 4}$. $^{\text {a }}$

\begin{tabular}{|c|c|c|c|c|c|c|c|c|c|}
\hline \multirow[b]{3}{*}{ Styren } & \multirow[b]{3}{*}{ HOMO } & \multirow[b]{3}{*}{$\alpha-$} & & & & \multicolumn{4}{|c|}{ Energy of SEO $\left(\mathrm{kcal} \mathrm{mol}^{-1}\right)$} \\
\hline & & & \multicolumn{3}{|c|}{ MO energies $(\mathrm{eV})^{\mathrm{b}}$} & \multicolumn{2}{|c|}{ PIDA $_{\text {HFIP }}$} & \multicolumn{2}{|c|}{$\mathbf{D M P}_{\text {HFIP }}$} \\
\hline & & & $\beta$-LUMO & $\mathrm{E}_{\mathrm{g}}^{\mathrm{c}}$ & $\mathrm{E}_{\mathrm{g}}^{\mathrm{d}}$ & $\Delta \mathrm{G}_{\mathrm{r}}$ & $\Delta \mathrm{G}^{\dagger}$ & $\Delta \mathrm{G}_{\mathrm{r}}$ & $\Delta \mathrm{G}^{\ddagger}$ \\
\hline 14 & -7.27 & -12.22 & -6.83 & 6.54 & 5.71 & 28.6 & 31.6 & 13.5 & 19.9 \\
\hline 15 & -7.18 & -11.33 & -6.06 & 6.45 & 5.62 & 20.3 & 30.7 & 6.6 & 21.2 \\
\hline 16 & -7.04 & -11.87 & -6.45 & 6.31 & 5.48 & 24.8 & 28.8 & 10.5 & 18.0 \\
\hline 17 & -7.28 & -12.11 & -6.73 & 6.55 & 5.72 & 27.1 & 30.5 & 12.7 & 19.5 \\
\hline 18 & -7.41 & -12.34 & -6.95 & 6.68 & 5.85 & 34.5 & 35.4 & 20.1 & 23.1 \\
\hline 19 & -7.69 & -12.33 & -7.01 & 6.96 & 6.13 & 31.6 & 34.0 & 17.3 & 22.4 \\
\hline 20 & -7.44 & -12.03 & -6.96 & 6.71 & 5.88 & 54.0 & 37.5 & 21.5 & 25.5 \\
\hline 21 & -7.53 & -12.63 & -7.11 & 6.80 & 5.97 & 31.1 & 33.4 & 16.8 & 21.8 \\
\hline 22 & -7.39 & -12.53 & -7.00 & 6.66 & 5.83 & 31.4 & 33.6 & 17.1 & 22.0 \\
\hline 23 & -7.79 & -13.21 & -7.54 & 7.06 & 6.23 & 36.9 & 38.1 & 22.6 & 25.8 \\
\hline 24 & -7.96 & -12.82 & -7.59 & 7.23 & 6.40 & 39.7 & 40.1 & 25.4 & 27.1 \\
\hline
\end{tabular}

${ }^{a}$ The calculations were carried out with hydrogen-bonded iodine reagents. ${ }^{\mathrm{b}}$ Calculations were performed in gas phase with $w$ B97XD/6-31G(d)/LANL2DZ level of theory, and the LUMO of DMP $\mathbf{P H F I P}_{\text {is }}-1.56 \mathrm{eV}$ and

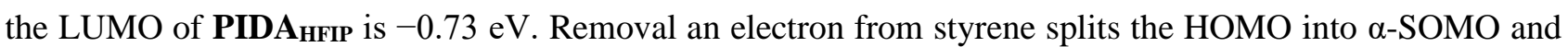
$\beta$-LUMO. ${ }^{\mathrm{c}}$ Energy gap $\left(\mathrm{E}_{\mathrm{g}}\right)=\mathrm{LUMO}\left[\mathbf{P I D A}_{\mathbf{H F I P}}\right]-\mathrm{HOMO}[$ styrene $] .{ }^{\mathrm{d}}$ Energy gap $\left(\mathrm{E}_{\mathrm{g}}\right)=\mathrm{LUMO}\left[\mathbf{D M P}_{\mathbf{H F I P}}\right]$ $-\mathrm{HOMO}[\mathrm{styrene}]$. 


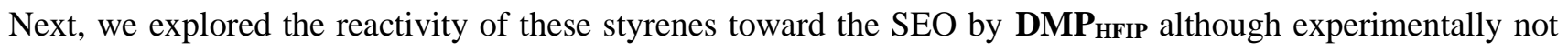
reported (Table 1). Expectedly, as we have seen above that iodine (V) is more reactive toward SEO, DMP $\mathbf{P F I P}_{\text {}}$

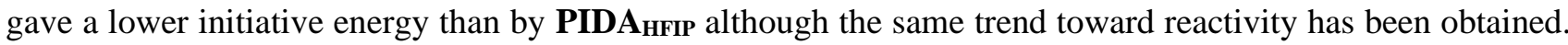

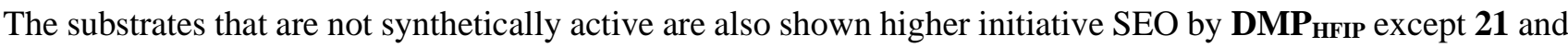
22. The free energy of activation for SEO by DMP $\mathbf{P F I P}_{\text {is }}$ significantly lower than for that obtained with PIDA $_{\text {HFIP }}$ even for those who are not reactive toward cycloaddition. The energy gaps $\left(\mathrm{E}_{\mathrm{g}}{ }^{\mathrm{c}}=\mathrm{LUMO}\left[\mathrm{DMP} \mathbf{P}_{\mathrm{HFIP}}\right]\right.$ - HOMO[styrene]) calculated with $\mathbf{D M P}_{\text {HFIP }}$ is lower than from those calculated for PIDA $\mathbf{A}_{\text {HFIP }}$ by $0.83 \mathrm{eV}$.

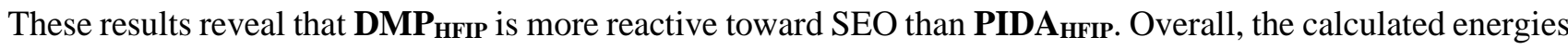
gave a good insight into reactivity of these styrenes toward cycloaddition and will raise a new understanding toward further attentions to advance further investigations.

\section{Conclusions}

DFT calculations at the (SMD)- wB97XD/Def2-TZVPP,6-311+G(d,p)//wB97XD/6-31G(d),LANL2DZ level of theory were exploited to provide mechanistic insights into the HVIR-promoted hetero- and homodimerizations of styrenes facilitated by HFIP. The computational level was validated through comparison between calculated and measured redox potentials of different substituted trans- $\beta$-methylstyrenes. The findings achieved in this study can be summarized as follows.

1- The HFIP molecules lower the energy of the single electron oxidation (SEO) or initiation as a result of strong hydrogen bonding interactions that substantially lower the energy of the frontier orbitals before and after electron addition.

2- The mechanism of HETD and HOMD is a radically-characterized $\pi$ - $\pi$ stacked head-to-head stepwise $[2+2]$ cycloaddition initiated via SEO by DMP and PIDA, respectively. DFT results supported by quasiclassical molecular dynamics simulations show that HOMD is a competing pathway to HETD although the latter is relatively faster, in accordance with experimental observations.

3- The initiation is a rate-determining step as a thermodynamically endergonic and propagation is accomplished by radically-cationic hetero- and homodimerized intermediates and propagation is faster than termination by radically-anionic HVIRs.

4- Initiation by $\mathbf{D M P} \mathbf{P}_{\text {HFIP }}$ found to be faster and thermodynamically more favorable than by PIDA $\mathbf{A}_{\mathbf{H F I P}}$ due to (1) the SOMO energy of the radical anion $\mathbf{D M P}^{-}{ }_{\text {HFIP }}$ is lower than $\mathbf{P I D A}^{-}{ }_{\text {HFIP }}$ and (2) the calculated energy gap of electron transfer for a SEO ( $\mathrm{E}_{\mathrm{g}}=\mathrm{LUMO}[\mathrm{HVIR}]-\mathrm{HOMO}\left[\right.$ styrene]) by $\mathbf{D M} \mathbf{P}_{\text {HFIP }}$ is lower than PIDA HFIP $_{\text {by }} 0.83 \mathrm{eV}$. 
5- Comparison between the measured and calculated redox potentials evaluated the important role of a $p$ methoxy group in the success of a styrene toward dimerization. We found that the SEO step that leads to a more thermodynamically favorable radical cation formation makes the cycloaddition more successful, indicating that the thermodynamic term represents a major contribution in the initiative barrier.

Overall, this mechanistic study brings significantly important insights into a such influential synthetic utility and opens possibilities toward advancing an efficient protocol for stereoselective approaches of simple and complex hetero- and homodimerizations. We envision that using DFT simulations on catalyzed SEO will enhance and warrant further attentions toward developing various oxidants to synthetically access a wide range of substrates used for bioactive and synthetic cyclobutane-containing products in a more efficiently-controlled fashion.

\section{Conflicts of interest}

There are no conflicts to declare.

\section{Acknowledgements}

The authors acknowledge the computational resources from the iridis4 supercomputer supported by the University of Southampton. A.A.H. highly acknowledge the University of Southampton/School of Chemistry for providing the visitor-status research position (2717441/EB00-VISIT). A.A.H. thanks Prof. Richard C. D. Brown for his valuable support.

\section{References}

1. Y. Xu, M. L. Conner and M. K. Brown, Angew. Chem. Int. Ed., 2015, 54, 11918-11928.

2. P. Margaretha, Helv. Chim. Acta, 2014, 97, 1027-1035.

3. V. M. Dembitsky, Phytomedicine, 2014, 21, 1559-1581.

4. V. M. Dembitsky, J. Nat. Med., 2008, 62, 1-33.

5. S. Cretton, T. A. Bartholomeusz, D. Jeannerat, O. Munoz, P. Christen and K. Hostettmann, Planta Med., 2009, 75, 916-916.

6. H. Ito, T. Toyoda and M. Sawamura, J. Am. Chem. Soc., 2010, 132, 5990-5992.

7. N. N. Noucti and E. J. Alexanian, Angew. Chem. Int. Ed., 2015, 54, 5447-5450. 
8. M. Gulías, A. Collado, B. Trillo, F. López, E. Oñate, M. A. Esteruelas and J. L. Mascareñas, J. Am. Chem. Soc., 2011, 133, 7660-7663.

9. M. R. Luzung, P. Mauleón and F. D. Toste, J. Am. Chem. Soc., 2007, 129, 12402-12403.

10. K. Chiba, T. Miura, S. Kim, Y. Kitano and M. Tada, J. Am. Chem. Soc., 2001, 123, 11314-11315.

11. B. B. Rath, G. K. Kole, S. A. Morris and J. J. Vittal, Chem. Comm., 2020.

12. A. Ledwith, Acc. Chem. Res., 1972, 5, 133-139.

13. F. A. Bell, R. A. Crellin, H. Fujii and A. Ledwith, J. Chem. Soc. D: Chem. Comm., 1969, 251-252.

14. N. L. Bauld, D. J. Bellville, B. Harirchian, K. T. Lorenz, R. A. Pabon, D. W. Reynolds, D. D. Wirth, H. S. Chiou and B. K. Marsh, Acc. Chem. Res., 1987, 20, 371-378.

15. N. L. Bauld, D. J. Bellville, R. Pabon, R. Chelsky and G. Green, J. Am. Chem. Soc., 1983, 105, 23782382.

16. N. L. Bauld and R. Pabon, J. Am. Chem. Soc., 1983, 105, 633-634.

17. M. A. Ischay, M. S. Ament and T. P. Yoon, Chem. Sci., 2012, 3, 2807-2811.

18. M. Riener and D. A. Nicewicz, Chem. Sci., 2013, 4, 2625-2629.

19. Y. Jiang, C. Wang, C. R. Rogers, M. S. Kodaimati and E. A. Weiss, Nat. Chem., 2019, 11, 1034-1040.

20. M. A. Ischay, Z. Lu and T. P. Yoon, J. Am. Chem. Soc., 2010, 132, 8572-8574.

21. Z. Lu and T. P. Yoon, Angew. Chem. Int. Ed., 2012, 51, 10329-10332.

22. A. E. Hurtley, Z. Lu and T. P. Yoon, Angew. Chem. Int. Ed., 2014, 53, 8991-8994.

23. S. A. Fleming and S. C. Ward, Tetrahedron Lett., 1992, 33, 1013-1016.

24. S. C. Ward and S. A. Fleming, J. Org. Chem., 1994, 59, 6476-6479.

25. T. Horibe, K. Katagiri and K. Ishihara, Adv. Syn. Catal., 2020, 362, 960-963.

26. L.-M. Zhao, T. Lei, R.-Z. Liao, H. Xiao, B. Chen, V. Ramamurthy, C.-H. Tung and L.-Z. Wu, J. Org. Chem., 2019, 84, 9257-9269.

27. R. Li, B. C. Ma, W. Huang, L. Wang, D. Wang, H. Lu, K. Landfester and K. A. I. Zhang, ACS Catalysis, 2017, 7, 3097-3101.

28. H. Amjaour, Z. Wang, M. Mabin, J. Puttkammer, S. Busch and Q. R. Chu, Chem. Comm., 2019, 55, 214-217.

29. A. Yoshimura and V. V. Zhdankin, Chem. Rev., 2016, 116, 3328-3435.

30. Y. Li, D. P. Hari, M. V. Vita and J. Waser, Angew. Chem. Int. Ed., 2016, 55, 4436-4454.

31. I. Colomer, A. E. R. Chamberlain, M. B. Haughey and T. J. Donohoe, Nat. Rev. Chem., 2017, 1, 0088.

32. Y. Zhu, I. Colomer, A. L. Thompson and T. J. Donohoe, J. Am. Chem. Soc., 2019, 141, 6489-6493.

33. I. Colomer, R. C. Barcelos, K. E. Christensen and T. J. Donohoe, Organic Letters, 2016, 18, 5880-5883.

34. I. T. Raheem, P. S. Thiara, E. A. Peterson and E. N. Jacobsen, J. Am. Chem. Soc., 2007, 129, 1340413405 .

35. H. Lv, J.-H. Zhan, Y.-B. Cai, Y. Yu, B. Wang and J.-L. Zhang, J. Am. Chem. Soc., 2012, 134, 1621616227.

36. A. G. Doyle and E. N. Jacobsen, Chem. Rev., 2007, 107, 5713-5743. 
37. K. C. Nicolaou, P. S. Baran, Y.-L. Zhong and J. A. Vega, Angew. Chem. Int. Ed., 2000, 39, 2525-2529.

38. K. C. Nicolaou, P. S. Baran, Y. L. Zhong, S. Barluenga, K. W. Hunt, R. Kranich and J. A. Vega, J. Am. Chem. Soc., 2002, 124, 2233-2244.

39. K. C. Nicolaou, P. S. Baran, R. Kranich, Y.-L. Zhong, K. Sugita and N. Zou, Angew. Chem. Int. Ed., 2001, 40, 202-206.

40. B. Janza and A. Studer, J. Org. Chem., 2005, 70, 6991-6994.

41. I. Colomer, R. Coura Barcelos and T. J. Donohoe, Angew. Chem. Int. Ed., 2016, 55, 4748-4752.

42. Y. Zhu, I. Colomer and T. J. Donohoe, Chem. Comm., 2019, 55, 10316-10319.

43. I. Colomer, C. Batchelor-McAuley, B. Odell, T. J. Donohoe and R. G. Compton, J. Am. Chem. Soc., 2016, 138, 8855-8861.

44. K. Farshadfar, A. Chipman, B. F. Yates and A. Ariafard, ACS Catalysis, 2019, 9, 6510-6521.

45. B. Ganji and A. Ariafard, Org. Biomol. Chem., 2019, 17, 3521-3528.

46. A. Kaur and A. Ariafard, Org. Biomol. Chem., 2020, 18, 1117-1129.

47. S. De Munari, M. Frigerio and M. Santagostino, J. Org. Chem., 1996, 61, 9272-9279.

48. L. L. O'Nei and O. Wiest, J. Org. Chem., 2006, 71, 8926-8933.

49. C. Guo, L. Cui, B. Chen, J. Yuan and Z. Tian, RSC Advances, 2012, 2, 9932-9937.

50. Y. Li, C. Guo and B.-Z. Chen, Comput. Theor. Chem., 2016, 1078, 163-172.

51. S. F. Nelsen, S. C. Blackstock and Y. Kim, J. Am. Chem. Soc., 1987, 109, 677-682.

52. V. Vaissier, P. Barnes, J. Kirkpatrick and J. Nelson, Phys. Chem. Chem. Phys., 2013, 15, 4804-4814.

53. F. Manke, J. M. Frost, V. Vaissier, J. Nelson and P. R. F. Barnes, Phys. Chem. Chem. Phys., 2015, 17, 7345-7354.

54. C. Demaille and A. J. Bard, Acta Chem. Scan., 1999, 53, 842-848.

55. E. R. Johnson, S. Keinan, P. Mori-Sánchez, J. Contreras-García, A. J. Cohen and W. Yang, J. Am. Chem. Soc., 2010, 132, 6498-6506.

56. J. M. Burns and E. D. Boittier, J. Org. Chem., 2019.

57. R. Villar López, O. N. Faza and C. Silva López, J. Org. Chem., 2017, 82, 4758-4765.

58. A. Patel, Z. Chen, Z. Y. Yang, O. Gutierrez, H. W. Liu, K. N. Houk and D. A. Singleton, J. Am. Chem. Soc., 2016, 138, 3631-3634.

59. L. Xu, C. E. Doubleday and K. N. Houk, J. Am. Chem. Soc., 2011, 133, 17848-17854.

60. L. Xu, C. E. Doubleday and K. N. Houk, J. Am. Chem. Soc., 2010, 132, 3029-3037.

61. K. Black, P. Liu, L. Xu, C. Doubleday and K. N. Houk, Proc. Natl. Acad. Sci. U.S.A., 2012, 109, 1286012865 .

62. A. Patel, Z. Chen, Z. Yang, O. Gutiérrez, H.-w. Liu, K. N. Houk and D. A. Singleton, J. Am. Chem. Soc., 2016, 138, 3631-3634.

63. J.-D. Chai and M. Head-Gordon, Phys. Chem. Chem. Phys., 2008, 10, 6615-6620. 